\title{
Research and Enlightenment of International Working Memory Based on Knowledge Mapping
}

\author{
Wang Yulan ${ }^{1, *}$, Deng Guomin ${ }^{2}$ \\ ${ }^{1}$ Department of Philosophy, Nanjing University, Nanjing, China \\ ${ }^{2}$ College of Education Science, Guiyang University, Guiyang, China
}

\section{Email address:}

yulan_flower@163.com (Wang Yulan),dam1981@126.com (Deng Guomin)

${ }^{*}$ Corresponding author

\section{To cite this article:}

Wang Yulan, Deng Guomin. Research and Enlightenment of International Working Memory Based on Knowledge Mapping. Science Innovation. Vol. 8, No. 6, 2020, pp. 182-193. doi: 10.11648/j.si.20200806.15

Received: January 15, 2021; Accepted: January 29, 2021; Published: January 30, 2021

\begin{abstract}
Working memory is the theoretical basis of cognitive load theory and multimedia learning theory. It provides a basis for us to understand the nature and process of learning, design and develop learning resource environment, and develop and construct new learning theories. In this paper, CiteSpace software is used to analyze the knowledge map and content of internationally important working memory research literature data, to reveal the current research status and future development trends of international working memory. The study found that the research of working memory originated from the discussion of human memory structure, and think it is not comprehensive to use short-term memory to describe the temporary storage of information by people, for it also involves the manipulation and processing of information, and the concept of working memory is proposed. In the past 20 years, working memory research has focused on seven aspects: disease damage, cognitive control, ability performance, individual differences, memory models, experimental methods and brain activation. Finally, the paper discusses the current limitations of working memory research, and discusses some issues that need to be focused on in the future of working memory research, and its implications for the design and development of instructional design principles, learning theory construction and learning environment.
\end{abstract}

Keywords: Working Memory, Learning Theory, Cognitive Impairment, fMRI, CiteSpace, Knowledge Map

\section{基于知识图谱的国际工作记忆研究及启示}

汪玉兰 $^{*}$ ，邓国民 ${ }^{2}$

1南京大学哲学系, 南京, 中国

2贵阳学院教育科学学院, 贵阳, 中国

邮箱

yulan_flower@163.com（汪玉兰），dam1981@126.com（邓国民）

摘要: 工作记忆是认知负荷理论和多媒体学习理论的理论基础, 为我们理解学习的本质和发生的过程、设计和开发学 习资源环境以及发展和构建新的学习理论提供了依据。本文使用CiteSpace软件, 对国际上重要的工作记忆研究文献数 据进行知识图谱及内容分析, 揭示国际工作记忆的研究现状和未来发展趋势。研究发现, 工作记忆研究源于对人的记 忆结构的探讨, 认为用短时记忆来描述人对信息的暂时存储是不全面的, 因为它还涉及到对信息进行操控与处理, 于 是提出了工作记忆的概念。近 20 年来工作记忆研究主要围绕疾病缺失、认知控制、能力绩效、个别差异、记忆模型、 实验方法和大脑激活等七个方面的内容展开。论文最后讨论了工作记忆研究目前的局限性, 并探讨了工作记忆研究未 来需要重点关注的一些问题, 及其对于教学设计原则、学习理论建构和学习环境的设计开发可能的启示意义。 
关键词: 工作记忆, 学习理论, 认知障碍, fMRI, CiteSpace, 知识图谱

\section{1. 引言}

工作记忆是认知负荷理论和多媒体学习理论的理论 基础, 为我们理解学习的本质和发生的过程、设计和开发 学习资源环境以及发展和构建新的学习理论提供了依据。 本研究对国际上主要的工作记忆研究文献数据进行知识 图谱及内容分析, 揭示其近二十年来的发展演进过程、阐 明工作记忆研究的最新发展, 并对其未来发展趋势进行展 望, 以期为工作记忆的进一步研究及其教学实践应用, 以 及信息化环境下的学习理论建构、应用和研究提供参考。

为了能够绘制系统全面的国际工作记忆研究知识图 谱, 本研究制定检索策略为 ( $\mathrm{TI}=$ ("working memory"), 索引=SCI-EXPANDED, SSCI, A\&HCI, 时间跨度=所有年 份), 从Web of Science核心数据库中检索到工作记忆研 究文献9082条, 将这些文献数据按“全记录与引用的参考 文献”记录内容导出用于后续分析, 这些文献的所有被引 文献共同构成它们的知识基础。本文使用CiteSpace软件对 工作记忆研究文献数据进行知识图谱及内容分析, 揭示该 研究领域的起源、发展脉络及未来趋势。

\section{2. 国际工作记忆研究的热门主题}

主题词（Term）和关键词（Keyword）是文献研究内 容的高度概括。本研究对国际工作记忆研究文献数据进行 了主题词和关键词共现分析, 绘制出国际工作记忆研究热 点知识图谱, 如图1所示。在该图谱中, 圆形结点表示关
键词, 方形结点表示主题词。可见, 国际工作记忆研究主 要涉及以下七个方面:

1. 疾病缺失 (A) 维度: 研究主题包括工作记忆损伤 与缺失、健康控制、老年痴呆症和精神分裂症等, 主要探 讨大脑神经方面的损伤、缺失与疾病对工作记忆带来的影 响。

2. 认知控制 (B) 维度: 主要探讨工作记忆的认知控 制机制及其影响因素; 研究主题有认知控制、认知功能、 智能、注意、选择性注意和认知等。

3. 能力绩效 $(\mathrm{C})$ 维度: 主要测量人的工作记忆能力 及其在工作记忆任务中的表现; 主要研究主题包括工作记 忆绩效、工作记忆能力、信息、维持、表征、工作记忆任 务和n-back任务等。

4. 个别差异 (D) 维度: 主要探讨工作记忆能力及表 现的个别差异及年龄差异; 具体研究主题包括个别差异、 儿童、老人和老年人等。

5. 记忆模型 (E) 维度: 主要探讨工作记忆理论模型 的构成与发展演化过程, 具体研究主题包括模型、短时记 忆、语言工作记忆、视觉工作记忆、执行功能和空间工作 记忆等。

6. 实验方法 (F) 维度: 重点讨论工作记忆研究使用 的主要研究方法, 具体研究主题包括干预、元分析、功能 性磁共振成像、控制组和正电子发射计算机断层显像等。

7. 大脑激活 $(\mathrm{G})$ 维度: 探讨工作记忆任务过程中对 大脑区域的激活, 并据此推导工作记忆功能对应的大脑皮 层区域, 具体包括前额叶皮层、激活、皮层、大脑等研究 主题。

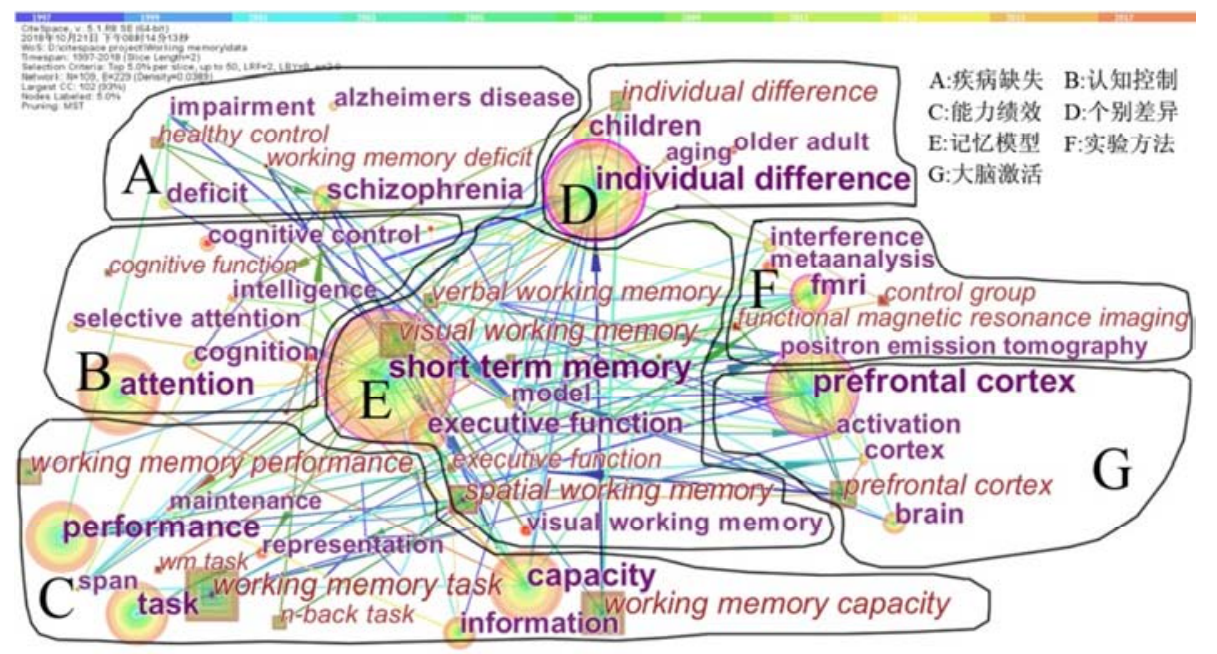

图1 国际工作记忆研究热点知识图谱。

\section{3. 工作记忆研究知识图谱分析}

\section{1. 重要文献分析}

利用CiteSpace软件对国际工作记忆研究文献数据进 行共被引分析（Cited Reference），生成工作记忆研究文
献共被引图谱, 如图2所示, 文献结点大小表示被引频次 多少, 外围紫色圆圈厚度表示文献结点中介中心性强度, 文献结点之间连线的粗细表示二者之间共被引现象的强 度, 颜色代表二者首次共被引发生的时间（参考上方时间 条颜色, 下同)。知识图谱能够将工作记忆研究领域的重 要研究文献 (包括高被引文献、高中介中心性文献和高突 
发性文献）及其共被引关系可视化表示出来，有助于我们 更好地理解工作记忆的重要概念、理论和原则, 把握近年 来研究的主要发展脉络, 并预测其未来的发展趋势。论文
将从国际工作记忆研究的七个主要研究领域, 对其重要文 献进行内容分析, 以揭示目前工作记忆七个研究维度的主 要研究进展。

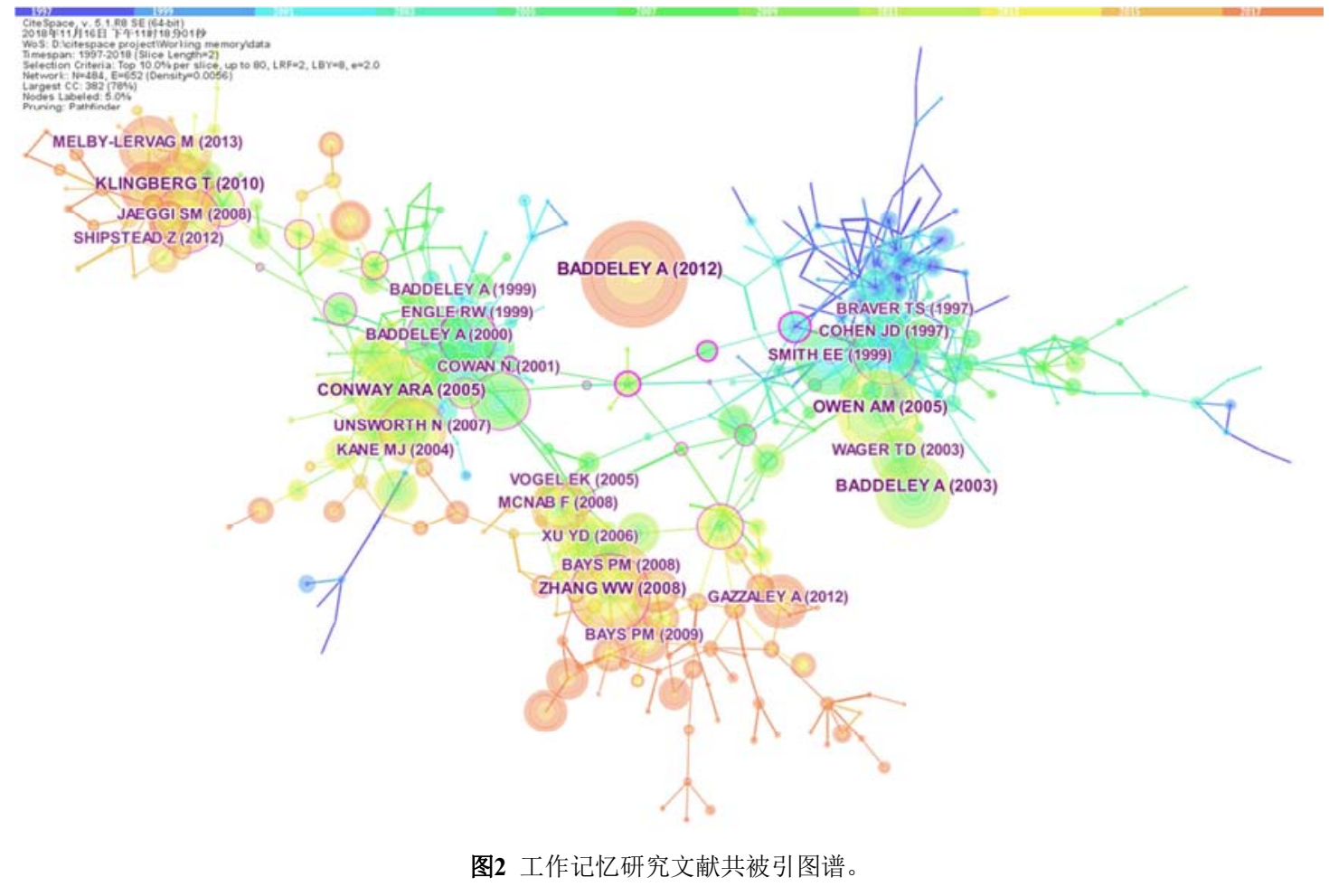

\subsection{1. 疾病缺失 (A) 维度的分析}

疾病缺失维度主要探讨大脑神经方面的损伤、缺失与 疾病对工作记忆及相关认知功能带来的影响。Manoach DS (1999) 使用功能磁共振成像技术进行实验研究发现, 精神分裂症患者在至少相等程度的右背外侧前额叶皮层 激活，显著更强的左背外侧前额叶皮层激活，以及背外侧 前额叶皮层激活的增强的空间异质性情况下, 表现出不良 的工作记忆表现。而且, 只有精神分裂症组激活基底神经 节（Basal Ganglia）和丘脑, 即使与正常组任务表现相匹 配也是如此。精神分裂症患组异常的工作记忆表现和大脑 激活可能反映出 WM 的前纹状体回路 (Frontostriatal Circuits）功能障碍。[1] Lee H（2017）指出工作记忆是精 神分裂症的核心认知缺陷, 过去的研究结果表明显著增加 的记忆刺激能够改善症状，但这种效果受到精确感知的影 响: 生物运动中包含的信息通常可以促进工作记忆编码, 但这种效应在精神分裂症案例中容易受到认知负荷增加 的影响。[2] Matsuzaka C T (2017) 通过实验证明了儿茶 酚-O-甲基转移酶 (COMT) 基因的功能性单核苷酸多态 性（SNP）可以介导前额叶皮层中认知与多巴胺活性之间 的关系, 而精神分裂症的核心特征是PFC中的多巴胺功能 障碍引发的认知障碍。[3] Nielsen J D （2017）指出工作 记忆损伤是精神分裂症的核心方面, 并使用实验研究提供 了精神分裂症早期阶段受到前顶叶皮层有效连接的工作 记忆调节受损的证据, 即使工作记忆表现正常, 也存在情 境敏感耦合失败。[4]

\subsection{2. 认知控制 (B) 维度的分析}

认知控制维度主要探讨工作记忆的信息表征、认知处 理过程机制以及与其他认知因素之间的关系等方面的问 题。关于视觉工作记忆中的信息表征及容量限制的性质等 问题, 一直以来争议较大, 有人认为工作记忆存储一组有 限的离散的、固定分辨率的表征, 还有人认为工作记忆由 一个资源池组成, 这些资源可以灵活分配，以提供少量的 高分辨率表征或者大量的低分辨率表征。Zhang WW(2008) 通过提供能力和分辨力的独立测量方法先后进行了 4 次测 量研究, 实验结果表明工作记忆存储观察对象子集的高分 辨率表征并且不保留关于其他对象的信息, 存储器分辨率 在很窄的范围内变化, 因此证明工作记忆存储并不是一般 的资源池, 而是一组离散的固定分辨率表征。[5]传统的观 点认为持续的、升高的活动负载敏感性是一种视觉短时记 忆中有限项目数量的存储指数。Emrich SM (2013) 使用 多体素模式分析来检查多个记忆负荷中人类VSTM的神 经表征, 研究发现VSTM的内容能够从对视觉刺激表现出 瞬时响应的区域解码, 但不能从持续的高负荷敏感性的延 迟期活动区域解码。而且, 存在于这些瞬时激活区域的神 经信息随着负荷的增加而显著降低, 表明支持VSTM维持 的活动模式的负荷敏感性。重要的是, 作为负荷功能的分 类性能的降低与助记符分辨率内的主体内变化相关。这些 研究结果表明, 感觉视觉皮层中神经活动的分布式模式支 持VSTM中信息的表征和精确性。[6] Bays PM（2008）显 示出视觉记忆容量不是由对象的数量来固定的, 而是在视 觉场景中的所有项目之间动态共享有限的资源。该资源可 
以在对象之间灵活地移动, 分配由于选择性注意而偏向于 即将到来的眼睛运动的目标。分配给每个项目的资源比例 决定了它被记住的精确度, 它们之间的关系由简单的幕律 控制, 允许对场景中的资源分布进行定量估计。[7]

工作记忆和选择性注意经常被视为两个独立的研究 主题。Awh E (2000) 发现空间工作记忆和空间选择性注 意机制之间存在功能重叠, 空间注意机制被招募用于类似 于维持工作记忆中活跃信息的复述功能, 使专门用于处理 真实空间的系统得到了很好的使用, 以便保持记忆空间的 活跃。未来还需要研究其他记忆系统, 确定感觉系统是否 更普遍地在记忆中发挥重要的作用。[8]Gazzaley A,(2012) 指出选择性注意力会影响工作记忆的表现, 实际上注意力 和工作记忆越来越被视为重叠的结构。人类神经生理学研 究的最新证据表明, 自上而下的调节作为这两种认知操作 的共同神经机制, 其核心特征包括刺激选择性感觉皮层中 的活动调节, 同时参与作为自上而下信息来源的前额叶和 顶叶控制区域。自上而下的调节同时在工作记忆任务的刺 激存在与缺失阶段进行, 比如期望记住的随之而来的刺激, 刺激的选择和编码, 维护相关信息和记忆检索。[9]

\subsection{3. 能力绩效 (C) 维度的分析}

能力绩效维度主要探讨人的工作记忆能力、测量、培 训及其在工作记忆任务中的表现。Cowan N（2001）对短 时记忆容量数量进行了重新思考, 通过大量的文献综述, 指出短时记忆容量只有 3 到 5 个。作者认为只有在可以明确 观察它们的边界条件时, 容量有限性才有助于分析信息处 理, 此时复述和长时记忆无法将刺激项组合成未知大小的 块。平均约四个块的容量限制与其他非容量限制源相关, 当单独保持的块的数量不清楚时, 以块表示的纯短时记忆 容量限制区别于复合的知识记忆限制。论文讨论了纯容量 估计值在一个狭窄范围内的原因, 并提出了注意集中能力 限制的观点。[10]Engle RW（1999）提出了一个工作记忆 和一般流体智能的理论, 认为工作记忆和流体智能反映了 保持表征活跃的能力, 特别是面对干扰和分心时, 并讨论 了这种能力和受控注意力的关系, 以及前额皮层的功能。 [11]

工作记忆的准确测量需要合适的具有一定信效度的 度量工具。Conway ARA（2005）对计算跨度、操作跨度 和读取跨度任务等工作记忆跨度任务作为测量工作记忆 容量度量工具的起源进行了综述, 指出这些跨度任务的底 层结构和实现方式实际上是相同的, 他们都强制工作记忆 去完成处理任务, 使其参与执行处理过程。论文同时讨论 了这些任务的信度、效度以及统计和分析技术等方面的问 题。[12] 这为我们使用跨度任务, 以及开发和分析新的任 务, 以更好地测量工作记忆和认知能力提供了启发。Kane MJ（2004）使用潜变量研究方法测试语言和视觉空间工 作记忆容量测量是否反映了一个主要领域的一般构造。通 过确认性因子分析和结构方程模型表明, 工作记忆任务在 很大程度上反映了领域一般因子, 工作记忆结构是流体智 力的强预测因子和领域具体推理的较弱预测因子, 研究结 果支持对工作记忆能力的领域一般的观点。[13]

虽然一般认为工作记忆的容量是有限的, 但一些新的 研究证据表明, 通过合适的训练可以改善工作记忆。
Olesen PJ (2004) 探讨了工作记忆训练引起的大脑活动的 变化, 通过实验研究发现经过训练后与额叶回中部和上下 顶叶皮层相关的大脑活动增强, 皮层活动的变化可以证明 工作记忆的基础--神经系统是可塑的。[14]Klingberg $\mathrm{T}$ （2010）指出通过适应性和扩展性培训可以改善工作记忆 的能力, 这种培训与额叶、顶叶皮层和基底神经节的大脑 活动变化, 以及多巴胺受体密度的变化有关。工作记忆训 练可以改善一般的工作记忆能力, 其效果能够转移到不同 材料类型和测试方式的非训练的工作记忆任务中。因此, 工作记忆训练可以作为一种注意力不集中等认知症状的 治疗干预措施, 适用于低工作记忆容量个体。[15] Jaeggi SM (2008) 讨论了流体智力对于知识推理和问题解决能 力和学生的重要性, 呈现了要求工作记忆任务的培训能够 促进流体智力的证据, 即使受过训练的任务与智力测验本 身完全不同, 并且证明了流体智力的改善程度受到训练量 的影响。[16]

\subsection{4. 个别差异 (D) 维度的分析}

个别差异维度主要探讨工作记忆能力及表现的个别 差异及年龄差异。Unsworth N（2007）对工作记忆容量个 体差异的研究进行了回顾, 发现工作记忆能力低者在各种 注意力和记忆力方面表现受损。作者认为工作记忆有限主 要由 2 个组成部分产生, 一为动态关注组件 (主存储器); 一为概率线索依赖搜索组件 (辅助存储器)。论文用该框 架对之前关于工作记忆容量个体差异的研究进行了述评, 并基于该框架预测即时自由回忆的表现以检验新的证据, 认为工作记忆容量的个体差异主要是由于能够维持主存 储器中可访问信息的能力, 以及从辅助存储器中搜索信息 的能力导致的。但仍存在一些尚待解决的问题, 首先, 主 要记忆和次要记忆之间的关系, 二者之间在多大程度上是 相互独立的或是否依赖于更一般的机制? 其次, 关于工作 记忆的理论模型过于简单, 将主要记忆和次要记忆看成一 个静态的系统, 面对复杂跨度任务时显得过于简单, 因此 需要一个更加复杂的动态系统, 以揭示两种不同记忆构件 之间是如何协同工作以实现表征的发生和处理过程的。第 三, 记忆模型主要是描述性的, 缺乏其所依据的某些模型 定量的严谨性, 因此未来的研究需要将个体差异纳入正式 模型, 以更好的计算现有数据。最后, 作者提倡整合综合 实验和差异研究的优势, 更细致地考虑个体在注意力和记 忆过程中的差异及其与高阶认知的关系。 [17] Wiemers E A (2018) 对工作记忆容量个体差异与动态认知控制之间 的关系进行了检查, 研究结果与认知控制理论和目标维持 理论的双重机制相符合, 阐明了工作记忆的个体差异是如 何表现为认知控制中的个体差异的。[18] Robison M K

（2017）探讨了工作记忆容量个体差异与注意力捕获易感 性之间的关系是否随搜索模式的变化而变化, 发现与工作 记忆容量相关的长时记忆差异驱动了注意力捕获效果, 这 有助于我们进一步理解记忆和注意力相互作用的细微差 别。[19]

\subsection{5. 记忆模型 (E) 维度的分析}

记忆模型维度主要探讨工作记忆理论模型的构成与 发展演化过程。Baddeley A和Hitch于1974年提出了最初的 
工作记忆模型, 包括语音环路、视空间面板和中央处理单 元三部分, 并分别介绍了各部分的功能特点和作用, 讨论 了它们和长时记忆之间的关系, 并对初始的工作模型进一 步发展, 认为工作记忆由一系列需要临时激活的流式系统 代表, 长时记忆代表更永久的晶体技能与知识（Baddeley A，2012）。[20] Baddeley AD （1999）进一步发展了工 作记忆的多成分模型, 认为工作记忆包括中央执行单元、 语音环路和视空间面板等多个专门的认知组成部分, 使人 们能够理解和心理表征他们的直接环境, 保留他们过去的 直接经验信息, 支持新知识的获取、解决问题, 以及制定、 联系和采取当前目标。该模型使用一系列实验方法, 为解 释有关工作记忆的各种实证研究结果提供了一个有用的 框架。[21]

Baddeley A（2000）对工作记忆三成分模型进行了回 顾, 认为它为来自正常成年人的数据, 以及神经心理学、 发展心理学和神经影像数据都提供了一种综合解释, 但是 还有一些现象仍无法捕获: 首先, 通过对语音环路的抑制, 发现虽然有明显的影响, 但不是毁灭性的, 而只是减少了 1 到 2 个数位; 其次, 证据表明视空间面板擅长存储单个复 杂模式, 而不适合串行调用。这些数据空间是如何存储的, 之前的工作记忆模型并没有提供这样的机制。研究数据表 明需要某种形式的后备存储, 能够支持连续调用, 并可能 整合了语音、视觉和其他类型的信息。第 3 , 简单的语音 环路假设回忆无意义的数据序列, 而实验证据表明, 临时 存储能够保存复杂的信息, 并能在远超工作记忆从属系统 假定的时间范围内使用它, 而这一功能在现有的构成中

（语音环路、视空间面板和中央处理单元）找不到合理的 解释。第 4 , 初始模型假设存储和复述过程是可分离的, 而研究证据表明假设某种形式的一般复述更为合理, 这可 能涉及对要回忆的材料的组成部分的连续关注。因此, 需 要对拟用的备用存储中的复述操作过程进行额外的假设, 在视空间面板中的复述需要类似的假设。第 5 , 该模型无 法存储两个从属系统以外的复杂图像, 它们在维持言语揭 示图像时起作用, 但并不似长时记忆的贡献那么重要, 这 再一次表明存在一个能从从属系统和长时记忆中获取信 息并以某种储存形式保存信息的备用记忆空间。基于这些 问题, 作者认为需要假设一种过程或机制, 用于将来自各 种子系统的信息协同地组合成一种临时的形式, 这种表征 还提供了对绑定问题和意识作用的可解决方案, 于是提出 了工作记忆的第四个构成一情境缓冲区，一个用于将各种 来源的信息集成到多维表征的系统, 如图3所示。情境缓 冲区被假设为一个容量有限的暂时存储系统, 能够整合不 同来源的信息, 由中央执行单元控制, 能够以意识的形式 提取信息, 为一系列系统提供了一个接口, 并使用多维编 码方式, 不仅提供用于环境建模的机制, 还提供用于创建 新的认知表征的机制, 这反过来可以促进问题的解决。[22]

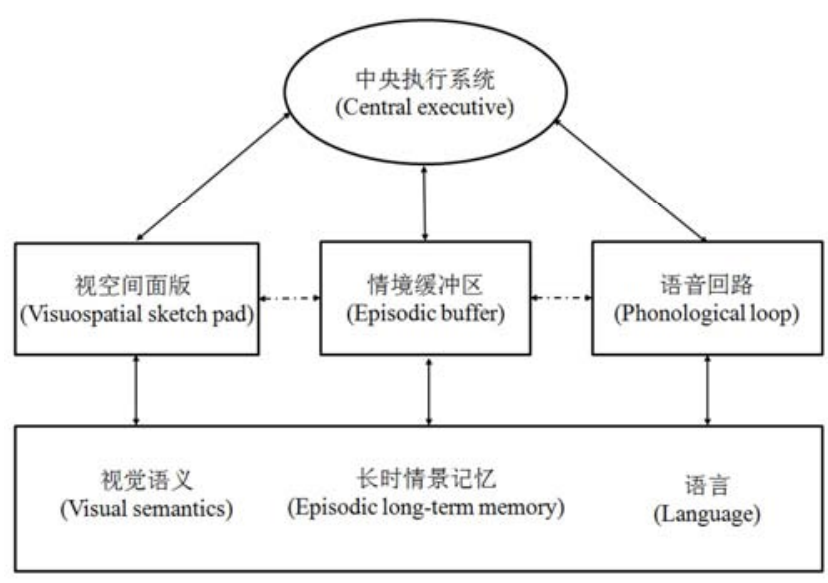

图3 多成分工作记忆模型。

Baddeley A（2003）以其工作记忆模型为基础对相关 文献进行了述评, 并对更为通用的工作记忆理论模型的未 来发展进行了推断。论文首先介绍了多成份工作记忆模型 的提出、构成、各构成部分的结构和功能特点, 以及整体 理论模型的发展过程和各方面仍需要进一步解决的问题。 然后, 论文对工作记忆解剖定位研究进行了综述, 发现工 作记忆的三个基本组成部分位于不同的脑区。论文最后讨 论了一个系统的工作记忆理论还需要探讨的其他方面: 1 . 需要在语音环路和语言研究与产生的工作之间, 以及空间、 视觉处理和动作控制的研究工作之间建立更紧密的联系; 2. 揭示工作记忆系统的驱动源和驱动机制是什么。3.揭示 工作记忆和不同控制层级之间的关系, 在更广泛的图景中 充分理解工作记忆的作用, 它注意力有限, 但是一个在人 的思想、计划和行为方面的一个至关重要的系统。[23] Baddeley A（2012）对保持相对稳定的整体理论框架以及 尝试在整体理论框架内构建更具体模型的尝试进行了区 分, 并讨论了替代模型及其与框架之间的关系 (如嵌入式 过程理论、个体差异理论、STM的思维与大脑模型、工作 记忆计算模型等), 并总结了对其进一步发展的猜想, 评 论了试图将模型和理论应用于它们通常基于的实验室研 究之外的价值。[20]

综上所述, Baddeley A的工作记忆多成分模型在工作 记忆研究领域产生了重要的学术影响, 而且在促进工作记 忆研究领域的知识交流过程中扮演了中心结点的作用。随 着认知神经科学、心理学和脑成像等方面研究的大量新的 证据的涌现, Baddeley A在过去的40多年间不断对其进行 修改和完善, 从最开始提出三成分模型, 到引入情境缓冲 区的四成分模型, 为不同研究方面的证据提供了一个得到 广泛认同的综合理论框架, 并且进一步揭示出工作记忆各 认知成分的运行机制、相互关系和信息表征方式, 以及工 作记忆与长时记忆和感觉登记等其他认知系统的关系。但 工作记忆模型在发展的同时, 也面临一些局限性, 一些学 者也对工作记忆理论模型未来的发展面临的挑战进行了 分析, 并提出了一些替代模型。Postle BR（2006）指出对 工作记忆的认知神经科学研究主要受到了心理学理论与 神经科学数据融合的标准模型的推动。该标准模型的原则 之一是工作记忆功能来自专门系统的操作, 这些系统充当 信息存储和操作的缓冲器, 其中额叶皮层, 尤其是前额叶 
皮层是这些专用系统的关键神经基质。但是标准模型同时 又成为其自身成功的牺牲品, 并且不再适应它所激发的许 多研究所产生的实证研究结果, 而且存在一些逻辑上的问 题。论文提出了一种替代方案, 认为工作记忆功能是通过 大脑系统的协同招募和注意而产生的, 大脑系统已经发展 出为实现感观、再生和行动相关的功能。来自猴子和人类 的行为、神经心理学、电生理学和神经影像学的研究证据, 被认为是如何解释前额皮层中的延迟期活动的问题。 [24]

\subsection{6. 实验方法 (F) 维度的分析}

工作记忆常用的研究方法有实验研究、元分析和神经 影像学等方法。实验研究将被试进行分组, 让他们参与不 同的工作记忆任务, 然后测量他们在这些任务中的表现或 使用神经影像学监测大脑皮层的激活, 并对测量数据进行 统计分析, 推论工作记忆过程中的信息处理、表征的具体 过程和特征, 以及不同大脑区域在工作记忆中承担的功能。 Manoach DS（1999）使用实验研究方法以及功能磁共振 成像技术揭示出精神分裂症患者与正常人脑激活区域的 差异。[1]Zhang WW（2008）使用实验研究方法证明视觉 工作记忆存储并不是一般的资源池, 而是一组离散的固定 分辨率表征。[5] Postle BR（2000）详细阐述了事件相关 fMRI技术的研究方法, 其关键特征背后的基本原理。论文 通过使用两种不同的实验, 描述了解决不同类型问题的方 法：1.使用分组分析证明了空间延迟期活动中, 尾状核中 的空间延迟期活动与运动系统的相互作用更大, 这种方法 通过折叠所有体现延迟期活动的体素, 以最大化分组分析 对实验诱导的激活变化的敏感性, 但并无法比较由尾状核 的地形可分离区域支持的空间和非空间记忆功能。2.使用 单一被试分析, 以解决地形-功能隔离假设, 因为这些包 括对延迟期活动中刺激物质的主要影响的测试, 以及依赖 于即将发生的运动突发事件的非空间延迟期活动的测试。 [25]

元分析主要通过对已有的工作记忆实验研究文献进 行二次统计分析, 综合考虑这些文献中使用的变量, 在它 们的数据讨论的基础上进行再次分析, 以更全面地揭示工 作记忆不同构成、材料、功能与脑区激活之间的关系, 或 者发现这些被分析文献因规模或单一视角的限制所忽略 的一些问题。Wager TD. (2003) 对60种工作记忆的神经 影像学研究进行了元分析, 考虑了三种存储材料（空间、 语言和对象）、三种执行功能（工作记忆的持续更新、时 间序列记忆以及工作记忆的信息操作) 以及材料和执行功 能之间的相互作用。材料类型分析显示, 预期的空间和非 空间存储之间的背-腹分离在后皮层中, 而不是前额叶皮 层中。在语言的工作记忆中发现左前额优势的一些支持, 但仅适用于执行需求低的任务。对于空间工作记忆, 执行 需求增加了前额叶皮层的右侧化。需要执行处理的任务通 常比仅执行存储的任务产生更多的额叶背侧激活, 但并非 所有执行流程都显示这种模式。当需要不断更新工作记忆 和维持时间顺序的记忆时, 上额叶皮层 (superior frontal cortex）的Brodmann区域（BAs）6，8和9响应最多。在腹 侧额叶皮层（ventral frontal cortex）中，右侧BA10和47更 频繁地响应操纵需求 (包括双任务要求或心理操作)。后 顶叶皮层（posterior parietal cortex）中的BA7参与所有类
型的执行功能。作者最后考虑了一个潜在的第四个执行功 能, 选择性地关注存储在工作记忆中的刺激的特征, 这导 致在存储任务中激活内侧前额叶皮层（medial prefrontal cortex）（BA 32）的可能性增加。[26] Melby-lervag M, (2013) 对探讨工作记忆训练效果的随机对照组实验或准 实验研究的 23 项研究 30 组比较进行了元分析, 认为这些培 训计划在工作记忆技能方面产生了可靠的短期改善, 对于 言语工作记忆, 这些近期转移效应在随访时没有得到维持, 也没有足够的证据表明这种效应能够保持, 而且也没有令 人信服的证据表明工作记忆训练对其他技能的普遍化 (语 言和非语言能力、注意力抑制过程、词语解码和算法等)。 因此, 作者认为工作记忆训练计划似乎产生了不具有普遍 性的短期特定训练效果, 其作为儿童和健康成人认知功能 提升方法的效用让人怀疑。[27]

Owen AM (2005) 对使用n-back任务的工作记忆的功 能性神经影像学研究进行了元分析, 他选择了24项主要研 究, 从n-back变量操作过程 (位置和身份监测) 和工作记 忆内容 (言语和非言语) 两方面进行分析, 发现侧前运动 皮层、背扣带回和内侧前运动皮层、背外侧和腹外侧前额 叶皮层、额极、内侧和外侧后顶叶皮层等皮层区域被强有 力地激活。进一步辅以主要数据的适当子集的元分析, 证 明了语言刺激对于身份监测以及非言语刺激对于位置和 身份监测的广泛相似的激活模式。还有一些证据表明, 对 于不同的任务变量, 会有不同的额顶激活模式。作者对通 用大型额顶系统中每个主要皮层组件的功能特化进行了 讨论, 并指出定量元分析可以作为结合Talairach空间报告 中的多项初步研究结果的有力工具, 提供了不同版本的 n-back工作记忆范式广泛一致地激活额叶和顶叶皮层区域 的证据, 以及工作记忆对于过程和内容特定额顶激活的证 据。通过言语和非语言身份监测研究之间的正式比较, 发 现左侧腹外侧前额叶皮层、内侧和双侧前运动皮层、双侧 内侧后顶叶皮层和丘脑在言语任务时相对激活增强。非言 语刺激的位置和身份监测研究的比较表明, 右背外侧前额 叶和侧前运动皮层、右侧内侧顶叶皮层在位置监测任务时 激活相对较强, 而背扣带回、内侧前运动皮层在身份监测 活动时激活相对增强。论文最后逐一讨论了背外侧前额叶 皮层、腹外侧前额叶皮层、延髓头部前额皮层或前柱、双 侧和内侧前运动皮层和双侧与内侧后顶叶皮层等大脑区 域在工作记忆中起到的具体作用。[28]

\subsection{7. 大脑激活 ( $G$ ) 维度的分析}

大脑激活维度主要探讨工作记忆任务过程中对大脑 区域的激活, 并据此推导工作记忆功能对应的大脑皮层区 域。Braver TS. (1997) 使用功能磁共振成像（fMRI）探 测序列字母任务期间的前额叶皮层 (prefrontal cortex, PFC) 活动, 其中记忆负荷以递增的方式变化, 确定了 $\mathrm{PFC}$ 的背 外侧和左下部区域表现出的活动与工作记忆负荷之间的 线性关系, 同时使用全脑成像技术, 确定了其他大脑区域 与记忆负荷之间的线性关系, 建议一个参与PFC的分布。 [29]Cohen JD（1997）指出工作记忆负责存储短时存储和 在线操作高级认知功能所需要的信息, 如语言、计划和解 决问题。工作记忆一般认为包括执行过程和主动维持两种 类型的过程, 它们可能通过不同皮层结构来保留, 其中前 
额叶皮层容纳执行过程, 而更多的后部区域负责主动维护 内容特定缓冲区。但从对非人类灵长类动物的研究表明, 前额皮层的背外侧区域也可能参与主动维持。作者使用功 能磁共振成像检查执行工作记忆任务期间人类受试者的 大脑激活, 通过时间分辨率来检查区域激活的动态, 结果 显示前额叶皮层和顶叶皮层似乎在主动维持中起作用。 [30]

Courtney SM（1998）指出工作记忆是维持信息主动 表征的过程, 以便信息可以使用。猴子对空间工作记忆重 要的前额皮层区域位于主要的沟槽内和周围, 但在人类中, 空间工作记忆区域的位置, 甚至是否存在都存在争议。论 文通过使用功能性磁共振成像, 识别出额上沟区域专门负 责工作记忆, 这个区域相对于猴子大脑, 更靠上方和后方, 因此之前没有识别到。这是因为灵长类动物大脑进化过程 中, 人类空间工作记忆区域和额叶眼已经被外侧前额叶皮 层的更下部和前部的扩张所取代, 这也使得人脑区域发生 了位移从而形成了新的区域, 专门用于空间视觉的外部视 觉区域在人脑的顶叶皮层中比猴子具有更高的位置, 而专 门用于物体视觉区域在题叶皮层中具有更低的位置。两组 视觉区域的位移可能与系统发育上较新的皮层区域如 BA39和BA40等调节语言的出现有关。背侧额叶皮层中功 能区域的特定位移可能同样与其他认知能力的出现有关, 这些认知能力在人类中是独特的 (如抽象推理、复杂问题 解决和未来规划等), 或者是由前额叶皮层中的新功能区 域介导的。[31]

Smith EE（1999）对人的前额叶皮层中的存储和执行 过程进行了探测。前额叶皮层在工作记忆的调节过程中起 到重要的作用。近期使用功能神经影像学对工作记忆的两 种成分进行了研究, 发现不同类型的信息会激活不同的前 额叶区域, 言语材料的存储激活了布罗卡氏区、左半球补 充区和前运动区; 空间信息的存储激活了右半球前运动皮 层; 对象信息的存储激活了前额皮层的其他区域，这说明 前额叶皮层中的信息存储是模块化的, 空间信息表征比对 象信息表征更靠背后, 而且它是按过程组织的, 腹外侧区 域 (BA 45和BA 47) 调解维持存储所需要的操作, 背外 侧 (BA 46和 BA 9) 对存储中的信息进行主动操作, 言 语存储任务主要激活左半球语音区域, 空间存储激活右前 运动皮层, 对象存储激活PFC的更多腹侧区域, 仅需要存 储的语言任务的激活区域一般不会延伸到DLPFC, 而需要 执行流程和存储的口头任务会导致DLPFC在内的激活。两 个基本的执行过程是选择性注意和任务管理, 他们均激活 了前扣回和背外侧前额叶皮层。而且大量研究人员均认为 前额叶皮层调节执行过程, 并参与对工作记忆内容操作过 程的调节。执行过程包括注意\&抑制、任务管理、计划、 监测和编码等五种类型, 研究结果显示, 前扣带回可能与 认知冲突处理有关, 双任务激活了正面区域, 包括背外侧 前额叶皮层、前扣带回。[32]

Labar KS（1999）发现关于工作记忆和视觉空间注意 力的研究中都报道了前额叶和后顶叶激活, 为了直接比较 这两种认知功能所涉及的大脑区域, 让同一组受试者在进 行功能性磁共振成像时连续参与工作记忆和空间注意力 任务, 通过认知联合分析, 这两项任务共同的激活组包括 顶内沟、腹前中央沟、辅助运动区、额叶眼、丘脑、小脑、
左侧题叶皮层和右侧脑岛。双减法分析产生的额外的激活, 可以归因于前运动皮层、左下前额叶皮层、右下枯叶、楔 前叶和右小脑的言语工作记忆。枕题交界和纹状体外皮层 的额外激活可归因于隐蔽空间注意力。在同一受试组中使 用两个不同的任务使我们能够提供明确的证据, 即神经网 络在几个额顶点上保留空间注意力和工作记忆。因此, 他 们的发现支持主要的认知领域由部分重叠的大规模神经 网络表征, 这种重叠的存在表明空间注意力和工作记忆共 享与注意力资源的动态转移相关的共同认知特征。[33]

Rowe JB（2000）指出背外侧前额叶皮层是否参与维 持工作记忆中的条目或选择反应是有争议的, 作者使用事 件相关的功能磁共振成像研究人类对空间工作记忆任务 的表现, 他们将空间项目的维持与从记忆中选择项目进行 了区分, 以引导响应。研究发现选择但不是维持, 与背外 侧前额叶皮层的前额区域46的激活有关。相反, 维持与前 额叶区域8和顶内皮层的激活有关。研究结果支持背侧前 额叶皮层在表征选择中的作用, 这解释了当受试者在工作 记忆任务中的项目之间进行选择, 以及他们在意愿行动的 任务之间自由选择时, 该区域被激活的事实。[34]

Todd JJ (2004) 指出我们的视觉系统可以随时感知到 丰富而细致的视觉世界, 但我们只能记住视觉场景的一小 部分, 即我们对视觉场景的内在心理表征是非常稀疏的, 因为这种表征存储于一个容量有限的视觉短时记忆中

(Visual Short-term Memory, VSTM)。作者使用功能磁共 振成像技术, 显示出容量限制在大脑区域网络的一个结点 中被神经反映: 后顶叶皮层中的活动与可以存储在VSTM 中的有限量的场景信息紧密相关, 这表明后顶叶皮层是我 们视觉世界的贫乏心理表征的关键神经位置。论文通过一 系列实验, 证明在语言和视觉任务之间不存在交易, 即二 者之间是相对独立的, VSTM任务表示存在存储容量的限 制, 而不是空间参与显示或编码项的限制, 统计参数图揭 示了顶内和枕内沟中的单个双侧对称区域（IPS/IOS）的 峰值响应和编码的对象数量之间具有强相关性, 它们对视 觉场景的感知负荷不敏感, 对工作记忆的编码和维持阶段 敏感, 但对检索阶段不敏感, 它们涉及多种形式（对象身 份和位置) 的VSTM存储。此外, 作者还发现了前扣带回 (Anterior Cingulate, AC) 和腹枕区 (Ventral-Occipital, VO）在VSTM任务的感知和响应过程中被激活, 其中, 尤 其是 VO皮层, 体现了很强的规模效应, 说明它主要由感 知负荷而不是VSTM能够存储的信息量决定的。作者通过 一系列实验指出后顶叶皮层可能是视觉场景表征的容量 限制存储器的关键神经基础, 正面/前额皮层可能是整个 和维护该存储器所必需的, 特别是在延长的保留期间。由 于VSTM严重的存储容量限制, 后顶叶皮层对视觉世界的 表征可能是非常稀疏的。[35]

Curtis CE (2004) 指出工作记忆研究找到了神经证据, 即持续的神经元活动, 但对于这些活动实际记忆或编码的 内容并不清楚。论文在维持空间位置期间对人类大脑进行 了成像, 并且不管是否在延迟之前或之后选择了记忆引导 的扫视。眼动延迟匹配到样本任务用于测量维持的运动意 图, 因为已知整个延迟期间的即将到来的扫视方向。研究 使用了非匹配样本的任务, 其中扫视是不可预测的, 以测 量维持的空间注意力。在匹配延迟期间, 眼动神经区域更 
加活跃，而在非匹配延迟期间，后顶叶皮层和下额叶皮层 更加活跃。此外, 通过额叶眼的延迟周期活动来预测记忆 的保真度, 延迟期活动的太小与记忆引导的扫视的准确性 相关。通过实验控制反应选择, 可以在功能上分离额叶和 顶叶区域网络的结点, 这些结点通常在工作记忆研究中被 共同激活。因此可以推断出, 网络中不同结点保持不同的 表征代码、运动和空间, 哪些代码由持续的神经活动表征, 在从感知到行动的转换中选择响应。[36]

综上所述, 大量认知神经科学家综合利用脑成像和工 作记忆任务分析法, 分析不同工作记忆任务, 比如语言、 空间识别、对象识别和执行等不同任务激活的相应脑区, 揭示出工作记忆的语音回路、视空间面板和中央执行单元 等构成对应的脑区功能, 如图4所示。这也从神经物质基 础上证明了Baddeley工作记忆多成分模型的合理性，但关 于情境缓冲区, 以及工作记忆与长时记忆之间的关联性还 没有足够的神经影像学证据, 需要未来进一步的发展。

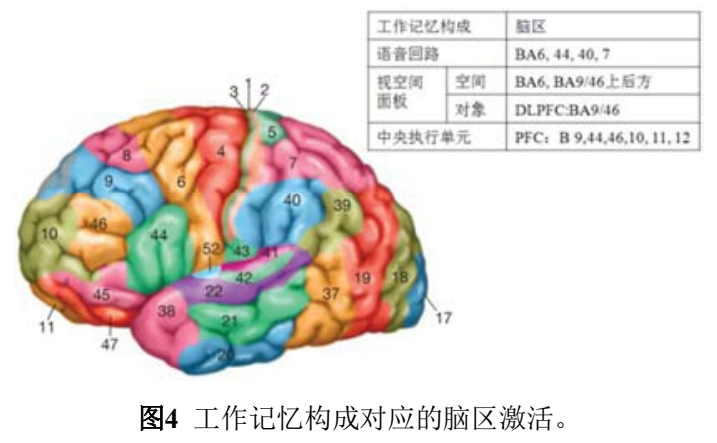

\section{2. 重要作者分析}

对工作记忆研究重要作者的共被引结构进行可视化 表征, 结果如图5所示, 其中引用频次超过1000次的作者 有Baddeley A. （Freq=4467）、Cowan N.（Freq=1794）、 Engle RW. (Freq=1418)、Wechsler D. (Freq=1353)、 Daneman M. (Freq=1177) 、Kane MJ. (Freq=1160)、Owen AM. (Freq=1112) 、Desposito M. (Freq=1078)、Smith EE. （Freq=1054）、Miyake A. (Freq=1039) 和Conway ARA. （Freq=1027）。Freq是指作者被引用的次数, 引用频次 较高的作者表明他们在工作记忆研究领域具有较大的学 术影响力。其中Baddeley A. 在过去二成分记忆模型的基础 上, 提出了工作记忆的多成分模型, 并在过去的数十年间 不断对其进行发展, 深入地研究了视觉工作记忆、语言工 作记忆、中央执行单元和情境缓冲区等不同构成的功能特 点。Cowan N在工作记忆的成熟与发展方面进行了大量的 研究, 对于认知增长的本质、工作记忆发展的特点及基础 以及注意力对工作记忆和认知能力中的作用等方面均作 出了重要的贡献。Engle RW. 探讨了工作记忆与执行注意 之间的关系, 并对工作记忆的个别差异进行了大量的实证 研究。Wechsler D. 在认知测量、成就测量和智力测量等方 面做出了巨大的贡献, 比如他设计和开发了个人成就测验 和智力测量量表, 为工作记忆能力、容量及其他认知能力 的测量提供了良好的工具支持。Daneman M. 对工作记忆在 语言学习与应用中的作用进行了研究, 具体研究主题包括 它对语言流畅性、阅读、理解、单词产生以及利用工作记
忆理论探讨阅读理解测试结构的有效性等方面。Kane MJ. 对工作记忆能力和执行注意力与思维控制之间的关系进 行了研究, 并分析了这些认知能力和大脑前额叶皮层之间 的关联性。Owen AM. 的研究领域主要集中在使用脑成像 技术, 揭示帕金森病人的工作记忆受损特点, 在工作记忆 对应的大脑区域方面做了大量的实验。Desposito M使用 fMRI技术研究了工作记忆空间工作记忆和非空间工作记 忆的信息维持与操作, 以及中央执行单元等构成的神经基 础。Smith EE使用神经影像学方法探测了空间与对象工作 记忆, 对言语和空间工作记忆进行了分离, 分析了言语工 作记忆的构成, 并揭示了前额叶皮层中的存储与执行过程。 Miyake A 讨论了执行功能个体差异的本质和组织, 分析了 工作记忆模型中的主动维持和执行控制机制, 以及这些功 能与前额叶皮层之间的关系。Conway ARA讨论了工作记 忆能力、短时记忆能力、信息速度和一般流体智力之间的 关系, 以及人类信息处理系统中记忆存储、选择性注意之 间的关系, 并提出了一个注意和记忆之间的整体框架。突 发性最强的 7 位作者分别是 Goldman-rakic PS.

（Burst=100.72）、Petrides M.（Burst=82.02）、Shipstead Z. (Burst=77.31)、Shallice T. (Burst=74.44)、 Mccarthy G. (Burst=70.69) 、Redick TS. (Burst=70.18) 和Paulesu E. （Burst=70.12）, 表明他们引领了近期工作记忆研究主题 的重要转向。Baddeley A. (Centrality $=0.44$ ) 、Luck SJ. (Centrality=0.26)、Smith EE. (Centrality $=0.24$ )、Cowan N. (Centrality $=0.19$ ) 和Awh E. (Centrality $=0.18$ ) 等5位作 者中介中心性最高, 表明他们在工作记忆研究的知识传播 网络中处于核心位置。
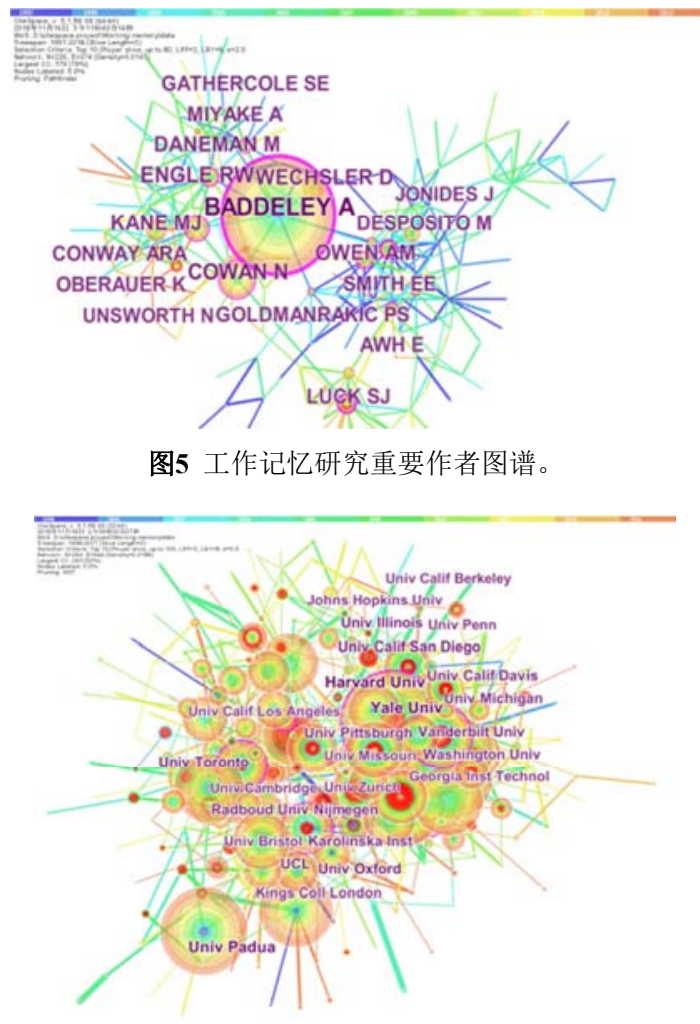

图6 工作记忆研究重要机构图谱。 


\section{3. 重要机构分析}

对工作记忆研究机构进行共现分析, 结果如图6所 示, 耶鲁大学 (Yale University)、哈佛大学 (Harvard University）、帕多瓦大学（University of Padua）、加 州大学圣迭戈分校 (University of California, San Diego）、牛津大学 (University of Oxford) 和多伦多 大学 (University of Toronto) 等机构具有较大的学术 影响力。突现率最高的五个研究机构分别为马克斯-普 朗克人类认知和脑科学研究所 (Max Planck Institute for Human Cognitive and Brain Sciences)、耶鲁大学

( Yale University) 、密歇根大学 (University of Michigan)、德克萨斯大学 (University of Texas) 和 加州大学伯克力分校 (University of California, Berkeley), 说明这些研究机构在工作记忆方面的研究 在近年来受到更广泛的关注。中介中心性最高的 5 个研 究机构分别是慕尼黑大学 (University of Munich) 、耶 鲁大学 (Yale University) 、密歇根大学 (University of Michigan)、剑桥大学 (University of Cambridge) 和 布里斯托大学 (University of Bristol), 说明他们在工 作记忆研究的知识传播过程中贡献较大。

\section{4. 重要出版物分析}

工作记忆研究出版物共被引分析结果如图7所示, 学术影响力最大的十本出版物分别为《Science》 (Freq=3991)、 《Proceedings of the National Academy of Sciences of the United States of America $\rangle$ ( Freq=3972)、

《The Journal of neuroscience》 ( Freq=3897) 、

《Neuropsychologia 》 ( Freq=3471) 、 《Nature 》

$($ Freq=3452 ) 、 《Trends in Cognitive Sciences 》

(Freq=3447) 、 《NeuroImage $)($ Freq $=3415)$ 、 《Journal OF Cognitive Neuroscience》 (Freq=3069) 、 《Cerebral Cortex》 ( Freq=3058) 和 《Journal of Experimental Psychology 》( Freq=3047)。近期最热门的出版物为 《Plos One》 ( Burst $=228.93$ ) 、 《Frontiers in Psychology》

(Burst $=227.84)$ 、 《Frontiers in Human Neuroscience》

(Burst=186.54) 、 《Working Memory》（Burst=179.1） 和 《Behavioral Neuroscience》（Burst=140.37）。中介 中心性最高的七本出版物分别为《Behavioral Neuroscience》 (Centrality $=0.49$ ) 、 《Trends in Cognitive Sciences 》 ( Centrality $=0.43$ ) 、 《 NeuroImage 》 (Centrality $=0.36$ ) 、 《Psychonomic Bulletin \& Review》

( Centrality $=0.27$ ) 、 《Psychological Science 》

( Centrality $=0.25$ ) 、 《Biological Psychiatr 》

( Centrality $=0.23$ ) 和 《 Journal of Experimental

Psychology》 （Centrality $=0.21 ） 。$

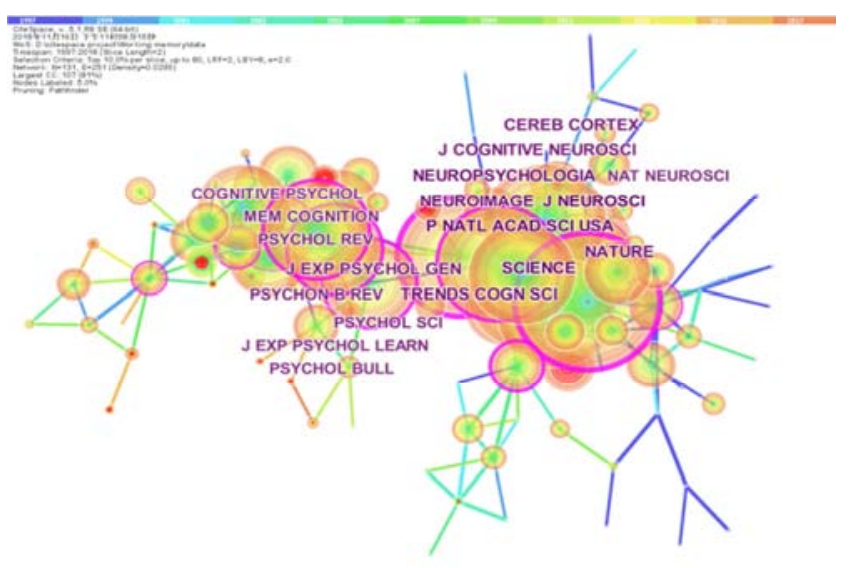

图7 工作记忆研究重要出版物图谱。

\section{5. 聚类分析}

在工作记忆研究文献共被引图谱的基础上进行聚类 分析, 结果如图8所示。可见, 关于工作记忆的研究聚类 包括个体差异 (\#0 individual differences) 、功能磁共振成 像 (\#1 functional magnetic resonance imaging) 、背外侧前 额叶皮层 (\#2 dorsolateral frontal cortex) 、前额叶皮层 (\#3 prefrontal cortex) 、视觉工作记忆（\#4 visual working memory) 、认知训练（\#5 cognitive training）、刷新（\#6 refreshing）、语音循环（\#7 phonological loop）、精神分 裂症 (\#8 schizophrenia)、神经振荡 (\#9 neural oscillations)、 学习困难 (\#10 learning difficulties)、精神运动兴奋剂 (\#13 psychomotor stimulant）、有限处理能力（\#14 limited processing capactiy) 和阅读障碍（\#15 dyslexia）等14个聚 类。可见, 功能性磁共振成像、背外侧前额叶皮层、学习 困难、精神运动兴奋剂和有限处理能力等聚类的文献在 2000年以前在共被引方面比较活跃, 个体差异、前额叶皮 层、语音环路、精神分裂和阅读障碍等聚类在 2000 年至 2010年期间共被引现象比较活跃, 2010年以后, 视觉工作 记忆、认知培训、刷新和神经振荡等聚类共被引现象活跃。 因此, 工作记忆早期的研究主要聚焦于对其容量有限性及 其与学习困难之间的关系, 并开始利用神经影像学研究工 作记忆对应的大脑区域, 再过渡到语音工作记忆成分与具 体能力障碍和疾病之间的关系, 在这一转换过程中, 文献 Cohen JD. (1997) 、Courtney SM. (1998) 、Labar KS. （1999）、Rowe JB. (2000)、Cowan N. (2001)、Awh E. (2001) 和Todd JJ. (2004) 等起到了衔接的作用。最 近几年, 对视觉工作记忆成分的研究进一步深入, 开始深 入挖掘工作记忆的运行机制, 并更加强调对之前理论研究 成果的实践应用, 比如对工作记忆能力的培训是否有助于 提高人的记忆能力和其他认知能力, 以及精神分裂症的治 疗等方面。在这一研究聚类的转换过程中, Postle BR. （2006）、Jaeggi SM.（2008）和Emrich SM.（2013）等 文献起到了核心衔接结点的作用。 


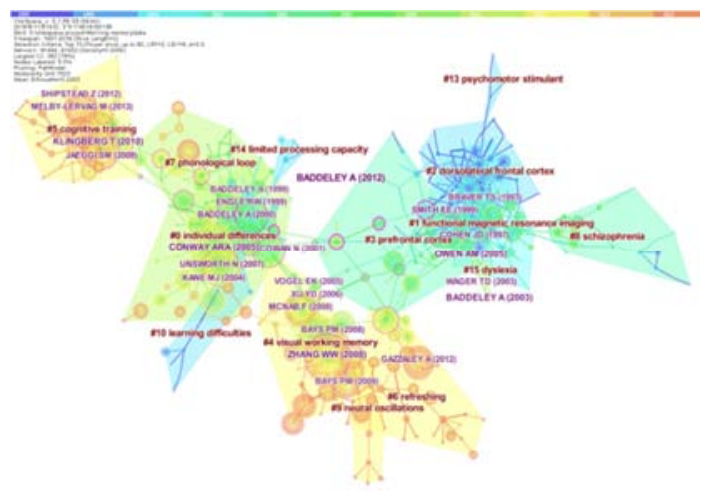

图8 基于关键词的聚类视图。
从聚类分析时间线视图来看（图9），关于个体差 异、前额叶皮层、视觉工作记忆、认知培训、语音环路 等研究聚类汇集了主要的高引用文献、高突现文献和高 中介中心性文献, 并且共被引现象活跃, 表明它们是工 作记忆的主要研究聚类。学习困难、有限处理能力、神 经运动兴奋剂和背外侧前额叶皮层等聚类的文献共被 引现象发生时间比较早, 后面逐渐消退, 说明它们是国 际工作记忆研究的问题缘起。最近的研究则主要以认知 培训、刷新和神经振荡等方面的聚类为主, 说明工作记 忆研究经过数十年的发展, 目前理论已经相对成熟, 将 来将在学习理论、认知能力提升等方面得到更多的实践 应用与检验。

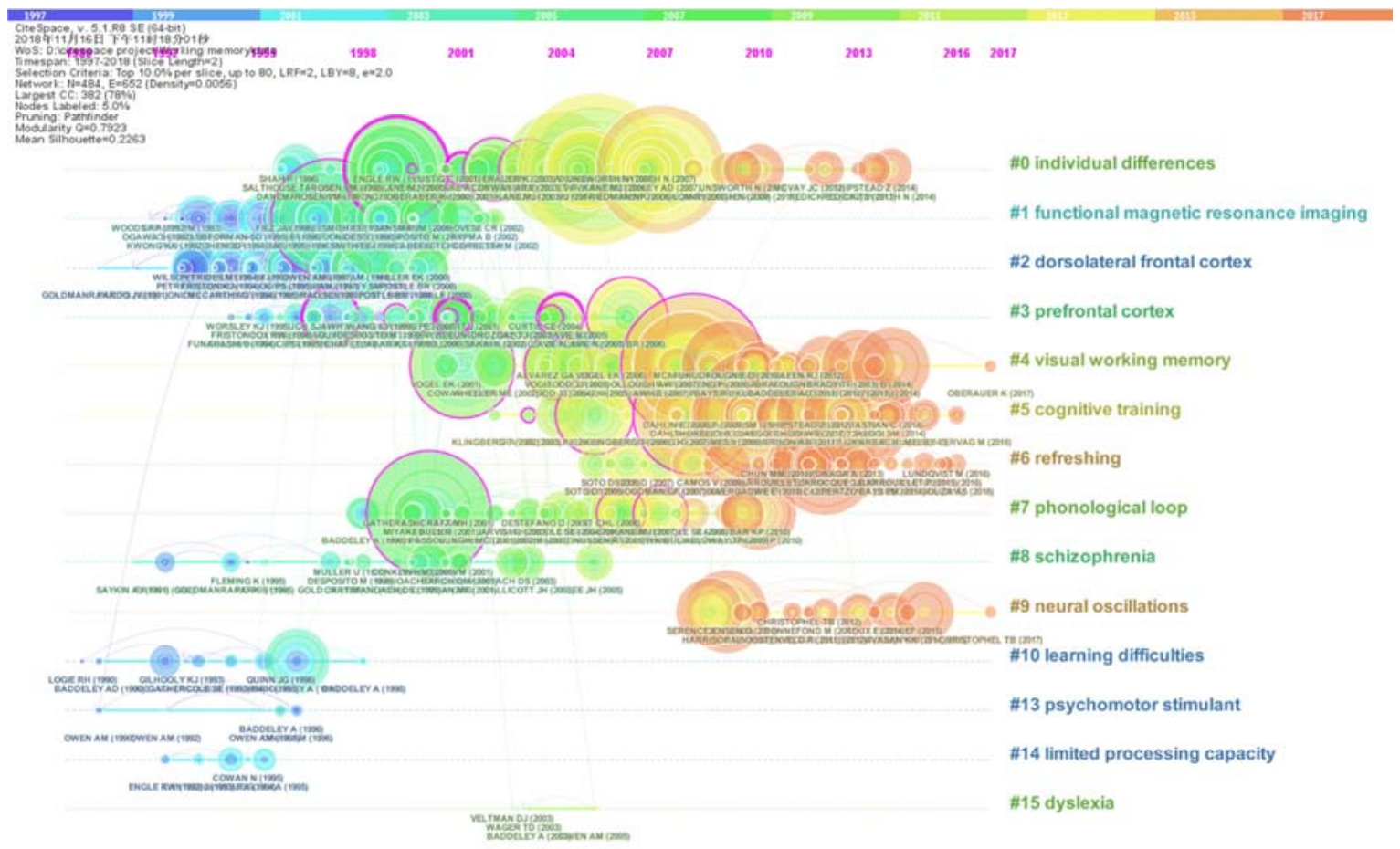

图9 聚类分析时间线视图。

\section{4. 研究发现}

工作记忆研究已经有半个多世纪的历史, 目前关于工 作记忆的理论模型、认知控制机制、能力测量与绩效及不 同功能成分对应的大脑区域等方面的研究均取得了较大 进展, 如何继续深入挖掘工作记忆理论的不足或者不清晰 的部分, 实现其进一步发展及其在学习和认知障碍治疗中 的实践应用是目前最需要关注的问题。使用CiteSapce软件 对国际上的工作记忆研究文献数据进行共被引和共现分 析, 绘制国际工作记忆研究知识图谱, 揭示其研究的起源、 发展脉络和未来趋势。

\section{1. 工作记忆研究起源}

工作记忆研究源于 20 世纪 60 年代中页认知心理学研 究领域对人类记忆系统结构的争论, 争论的焦点是记忆系 统结构是单一的还是一分为二的。随着研究的推进, 支持
二成分的证据越来越多。心理学家Atkinson和 Shiffrin于 1968年在《学习和动机心理学: 研究和理论进展》一文中 提出了二成分记忆系统模型, 将人类的记忆系统结构分为 长时记忆和短时记忆两种成分。70年代二成份记忆系统模 型也受到了挑战, 因为一些病例显示, 短时记忆受损的病 人表现出正常的长时记忆功能和很少的认知障碍。 Baddeley和 Hitch基于这样一个问题, 于1974年提出了记 忆系统结构的多成份模型, 认为工作记忆包括语音环路、 视空间面板和中央执行单元等三部分, 而且指出存储和处 理仅仅是工作记忆功能的一部分, 它的主要功能是协调记 忆系统的资源。随后, 这一工作记忆理论模型得到了越来 越多实验数据的支持, 在认知心理学、神经科学和学习领 域均产生了重要的影响, 而且激发了不同学科背景的人进 一步深入挖掘和发展工作记忆理论模型, 以及模型的构成 要素、认知操作及对应的大脑皮层区域, 发展了工作记忆 测量研究的能力测量方法和神经影像学技术, 并发展出一 些新的工作记忆模型。 


\section{2. 工作记忆研究发展脉络}

近年来, 工作记忆已经成为认知心理学、学习科学、 儿童发展等研究领域的重要理论基础, 多媒体学习和认知 负荷理论等都是建立在工作记忆研究基础之上的。工作记 忆在过去的研究与实践过程中, 演化出一些重要的研究领 域。首先, 工作记忆对其他认知能力和复杂认识任务的完 成均具有重要的影响, 一些临床病例, 比如老年痴呆症、 精神分裂症、抑有症以及学习和认知障碍等, 都和工作记 忆的缺失存在一定的关联性, 因此工作记忆成为这些认知 神经临床症状治疗的理论依据。其次, 在工作记忆理论模 型的基础上, 滋生了对不同类型工作记忆能力和容量进行 评价和测量的需求, 主要形成了两类测量研究方法与技术, 一种主要使用心理测量方法测量工作记忆的容量及其在 工作记忆任务中的表现, 如n-back任务、延迟匹配任务等。 另一种测量方法主要使用 fMRI等神经影像学技术测量工 作记忆任务期间大脑皮层的激活情况，从而找出不同工作 记忆任务对应的大脑区域, 目前工作记忆的主要构成、信 息类别和处理过程等功能已基本上找到了相应的脑区。第 三, 工作记忆除了信息的记忆存储功能, 还在执行其他认 知任务的过程中起到重要的协调作用, 关于它和其他认知 活动, 比如注意、长时记忆之间的关系, 以及工作记忆内 部各构成要素之间的关系，及其对不同类型信息的处理、 加工和表征方式也逐渐有了更新的认识。

\section{3. 工作记忆研究未来发展趋势}

尽管工作记忆研究在诸多领域均取得了不小的成绩, 但还有一些问题仍然需要未来更进一步深入的探讨。首先, Postle BR指出工作记忆模型引导了整个研究领域的方向, 促进了该领域的发展, 但它在得到越来越多实证数据支持 的同时, 又成为其自身成功的牺牲品：工作记忆模型强调 的是不同成分之间的分离, 但神经影像数据表明实际的信 息类型和处理过程要比想像的复杂的多, 很多任务对资源 的调用也是综合化的, 还且同时涉及到嗅觉、味觉和触觉 等感觉系统的征用, 因此, 工作记忆模型目前仍有发展的 空间。其次, 关于工作记忆的容量限制的原因和表征处理 机制, 目前也没有合理的解释, 有人用选择性注意力或注 意力资源集中来解释, 但目前仍缺少足够的实据。第三, 虽然神经影像学揭示出Baddeley\&Hitch模型构成的大致 对应脑区, 但其中的情境缓冲区还不是很确定, 而且很多 任务是由不同的区域之间形成的复杂的神经网络来完成 的, 因此各脑区之间是如何协同工作的, 还需要更复杂的 模型来演示和检验。神经影像学只是黑盒测试, 可以借用 更先进的计算模型和认知计算来实现工作记忆运行处理 过程的白盒测试，揭示其具体的执行和资源征用机制。第 四, 在工作记忆理论的基础上, 发展了认知负荷和多媒体 学习等著名的学习理论, 它们对教学设计和学习科学产生 了重要的影响, 近年来工作记忆研究也有了长足的发展, 未来需要加强在其最新研究成果的基础上, 设计更科学的 学习理论、认知技术和学习环境, 将其融入到在线学习环 境中的设计, 为学习者提供工作记忆和认知支架, 以更好 地支持学习者的学习。

\section{5. 结论}

本文使用CiteSpace软件, 对国际上工作记忆研究领域重 要研究文献、作者、机构和刊物进行共被引或共现分析, 并 绘制相关的知识图谱, 揭示出国际工作记忆研究的起源、发 展脉络和未来发展趋势。工作记忆源于对记忆进行分离, 即 开始将记忆分为长时记忆和短时记忆, 后来又发现工作记忆 的表述更为准确, 而且可以进一步细分为语音环路、视空间 面板和中央执行单元, 于是Baddeley等人提出了工作记忆的 多成分模型, 并在此基础上不断进行发展。工作记忆模型提 出来之后, 引起了认知心理学研究领域的持续关注, 而且得 到越来越多实证数据的支持, 其最初模型经过几次发展而日 益完善和合理, 并发展了自己独特的测量和实验研究方法, 验证了不同认认知构成的存在并找到了它们对应的脑区，而 且关于它们的认知控制机制也逐渐明了起来。近年来, 工作 记忆已经被广泛应用于精神分裂、老年痴呆、抑有症和认知 障碍等的临床治疗上, 而且衍生出大量的学习教学理论, 比 如认知负荷和多媒体学习理论等, 为教学设计和学习科学提 供了理论支持。但工作记忆模型仍然存在一些局限, 工作记 忆容量有限的原因和机理、情境缓冲区、不同功能构成之间 的关联机制还有待进一步明确, 同时需要在其最新研究进展 的基础上, 发展更多更合理的学习理论, 以指导教学设计、 学习环境和认知支架的设计, 达到提高教学效果的目的。

\section{致谢}

本文为教育部人文社会科学研究一般项目《基于学术 社交网络的高校科研影响力计量评价研究》(19YJA880005) 的阶段性成果之一。

\section{参考文献}

[1] Manoach DS, Gollub RL, Benson ES, et al. Schizophrenic subjects show aberrant fMRI activation of dorsolateral prefrontal cortex and basal ganglia during working memory performance. [J]. Biological Psychiatry, 2000, 48 (2): 99-109.

[2] Lee H, Kim J. Load-sensitive impairment of working memory for biological motion in schizophrenia [J]. PloS one, 2017, 12 (10): e0186498.

[3] Matsuzaka C T, Christofolini D, Ota V K, et al. Catechol-O-methyltransferase (COMT) polymorphisms modulate working memory in individuals with schizophrenia and healthy controls[J]. Revista Brasileira de Psiquiatria, 2017, 39 (4): 302-308.

[4] Nielsen J D, Madsen K H, Wang Z, et al. Working memory modulation of frontoparietal network connectivity in first-episode schizophrenia[J]. Cerebral Cortex, 2017, 27 (7): 3832-3841.

[5] Zhang W, Luck S J. Discrete fixed-resolution representations in visual working memory[J]. Nature, 2008, 453 (7192): 233.

[6] Emrich S M, Riggall A C, LaRocque J J, et al. Distributed patterns of activity in sensory cortex reflect the precision of multiple items maintained in visual short-term memory [J]. Journal of Neuroscience, 2013, 33 (15): 6516-6523. 
[7] Bays P M, Husain M. Dynamic shifts of limited working memory resources in human vision[J]. Science, 2008, 321 (5890): 851-854.

[8] Awh E, Jonides J. Overlapping mechanisms of attention and spatial working memory.[J]. Trends in Cognitive Sciences, 2001, 5 (3): 119-126.

[9] Gazzaley A, Nobre A C. Top-down modulation: bridging selective attention and working memory[J]. Trends in cognitive sciences, 2012, 16 (2): 129-135.

[10] Cowan N. The magical number 4 in short-term memory: a reconsideration of mental storage capacity[J]. Behavioral \& Brain Sciences, 2001, 24 (1): 87-114.

[11] Engle R W, Tuholski S W, Laughlin J E, et al. Working memory, short-term memory, and general fluid intelligence: a latent-variable approach [J]. Journal of Experimental Psychology General, 1999, 128 (3): 309-331.

[12] Conway A R A, Kane M J, Bunting M F, et al. Working memory span tasks: A methodological review and user's guide[J]. Psychonomic bulletin \& review, 2005, 12 (5): 769-786.

[13] Kane M J, Hambrick D Z, Tuholski S W, et al. The generality of working memory capacity: a latent-variable approach to verbal and visuospatial memory span and reasoning [J]. Journal of Experimental Psychology: General, 2004, 133 (2): 189.

[14] Olesen P J, Westerberg H, Klingberg T. Increased prefrontal and parietal activity after training of working memory.[J]. Nature Neuroscience, 2004, 7 (1): 75-79.

[15] Klingberg T. Training and plasticity of working memory[J]. Trends in cognitive sciences, 2010, 14 (7): 317-324.

[16] Jaeggi S M, Buschkuehl M, Jonides J, et al. Improving fluid intelligence with training on working memory [J]. Proceedings of the National Academy of Sciences, 2008, 105 (19): 6829-6833.

[17] Unsworth N, Engle R W. The nature of individual differences in working memory capacity: active maintenance in primary memory and controlled search from secondary memory[J]. Psychological review, 2007, 114 (1): 104.

[18] Wiemers E A, Redick T S. Working memory capacity and intra-individual variability of proactive control $[\mathrm{J}]$. Acta Psychologica, 2018, 182: 21-31.

[19] Robison M K, Unsworth N. Individual Differences in Working Memory Capacity Predict Learned Control Over Attentional Capture[J]. J Exp Psychol Hum Percept Perform, 2017, 43 (11): 1912.

[20] Baddeley A. Working memory: theories, models, and controversies[J]. Annual review of psychology, 2012, 63: 1-29.

[21] Baddeley AD, Logie RH (1999) Working memory: The multiple-component model. In: Miyake A, Shah P, eds. Models of working memory. Cambridge, UK: Cambridge University Press. 28-61.
[22] Baddeley A. The episodic buffer: a new component of working memory?[J]. Trends in cognitive sciences, 2000, 4 (11): 417-423.

[23] Baddeley A. Working memory: looking back and looking forward[J]. Nature reviews neuroscience, 2003, 4(10): 829.

[24] Postle BR. Working memory as an emergent property of the mind and brain.[J]. Neuroscience, 2006, 139 (1): 23-38.

[25] Postle BR, Zarahn E, D'Esposito M. Using event-related fMRI to assess delay-period activity during performance of spatial and nonspatial working memory tasks[J]. Brain Research Protocols, 2000, 5 (1): 57-66.

[26] Wager T D, Smith E E. Neuroimaging studies of working memory $[\mathrm{J}]$. Cognitive, Affective, \& Behavioral Neuroscience, 2003, 3 (4): 255-274.

[27] Melby-Lervåg $\mathrm{M}$, Hulme $\mathrm{C}$. Is working memory training effective? A meta-analytic review[J]. Developmental psychology, 2013, 49 (2): 270.

[28] Owen A M, McMillan K M, Laird A R, et al. N - back working memory paradigm: A meta - analysis of normative functional neuroimaging studies[J]. Human brain mapping, 2005, 25 (1): 46-59.

[29] Braver, Todd S., et al. "A parametric study of prefrontal cortex involvement in human working memory." Neuroimage 5.1 (1997): 49-62.

[30] Cohen J D, Perlstein W M, Braver T S, et al. Temporal dynamics of brain activation during a working memory task[J]. Nature, 1997, 386 (6625): 604.

[31] Courtney S M, Petit L, Maisog J M, et al. An area specialized for spatial working memory in human frontal cortex.[J]. Science, 1998, 279 (5355): 1347-1351.

[32] Smith E E, Jonides J. Storage and executive processes in the frontal lobes[J]. Science, 1999, 283 (5408): 1657-1661.

[33] LaBar, Kevin S., et al. "Neuroanatomic overlap of working memory and spatial attention networks: a functional MRI comparison within subjects." Neuroimage 10.6 (1999): 695-704.

[34] Rowe, James B., et al. "The prefrontal cortex: response selection or maintenance within working memory?." Science 288.5471 (2000): 1656-1660.

[35] Todd, J. Jay, and René Marois. "Capacity limit of visual short-term memory in human posterior parietal cortex." Nature 428.6984 (2004): 751.

[36] Curtis C E, Rao V Y, D'Esposito M. Maintenance of spatial and motor codes during oculomotor delayed response tasks[J]. Journal of Neuroscience the Official Journal of the Society for Neuroscience, 2004, 24 (16):3944. 